\title{
A Novel Classification and Minimally Invasive Treatment of Degenerative Lumbar Spinal Stenosis
}

\author{
Guangfei GU, Hailong ZHANG, Shisheng HE, Qingsong FU, Xiaobing CAI, Xu ZHOU, Xiaolong SHEN, Xin GU \\ Shanghai Tenth People's Hospital, Tongji University School of Medicine, Department of Orthopedics, Shanghai, China
}

\section{ABSTRACT}

\begin{abstract}
AIM: We conducted a novel classification system of degenerative lumbar spinal stenosis (DLSS) based on clinical manifestations and imaging (computed tomography and magnetic resonance imaging) features. We chose different minimally invasive surgical procedures according to our system. Clinical parameters and radiological findings will be assessed in the article.
\end{abstract}

MATERIAL and METHODS: A retrospective study was conducted on 96 patients undergoing minimally invasive surgery for DLSS. We chose different surgical procedures according to our novel classification system based on clinical manifestations, imaging features, and concurrence with other spinal diseases. Clinical parameters and radiological findings were assessed pre- and postoperatively.

RESULTS: The mean follow up period was 24 months (range, 15 36 months). There was a statistically significant improvement in the Visual Analogue Scale (VAS) score of low back pain and leg pain after surgery $(p<0.05)$. According to the Japanese Orthopaedic Association (JOA) scores, the operation efficacy was excellent in 57 cases, good in 36 cases, and fair in 3 cases. According to Bridwell's criterion, the fusion rate was $96 \%(48 / 50)$ in patients who underwent fusion surgery. There were no cages or pedicle screws related complications.

CONCLUSION: Minimally invasive surgical treatment of DLSS has satisfactory outcomes according to the novel classification, but further long-term, prospective, randomized controlled studies involving a larger study group are needed to validate the long-term efficacy.

KEYWORDS: Decompression, Degenerative lumbar spinal stenosis, Minimally invasive surgery, Transforaminal lumbar interbody fusion

\section{INTRODUCTION}

Spinal stenosis is a narrowing of the spinal canal with encroachment on the neural structures by surrounding bone and soft tissue (9). Patients with spinal stenosis typically present with intermittent neurogenic claudication, or a combination of lower extremity pain, tension and weakness that occurs with walking or standing but is relieved with sitting down or lumbar flexion. The pathoanatomy of degenerative lumbar spinal stenosis (DLSS) is very complicated, including bulging discs, zygapophyseal joint hypertrophy, buckling or hypertrophy of the ligaments, and spondylolisthesis as a result of the degenerative cascade (3). To our knowledge, how to do the individualized surgery for lumbar stenosis is still controversial. In this article, we conducted a novel classification system of DLSS based on clinical manifestations and imaging (Computed Tomography (CT) and Magnetic Resonance Imaging (MRI)) features. We chose different minimally invasive surgical procedures according to our system. Clinical parameters and radiological findings will be assessed in the article. 


\section{MATERIAL and METHODS}

\section{The Novel Classification System}

We used a novel classification system of DLSS based on clinical manifestations, and imaging (CT and MRI) features. According to the clinical manifestations, DLSS is divided into 3 types. Type I: intermittent claudication without leg pain. Type II: intermittent claudication with asymmetric radicular pain. Type III: intermittent claudication with bilateral radicular pain. According to the imaging (CT and MRI) features, DLSS is also divided into 3 types (Figure 1A-E). Type A: unilateral spinal stenosis (including lateral recess and nerve root canal) with or without central spinal stenosis. Type B: bilateral spinal stenosis (including lateral recess and nerve root canal) with or without central spinal stenosis. Type C: the other status, including lumbar spinal stenosis caused by calcification of huge lumbar disc herniation, severe DLSS and multiple-level ( $\geq 3)$ DLSS.

\section{Inclusion Criteria}

1) The clinical indications were leg pain and/or leg numbness inducing intermittent claudication rather than back pain,

2) Preoperative imaging examines including radiographs (anteroposterior, lateral and flexion-extension views), CT and MRI showed consistency with the clinical manifestations,
3) No obvious improvement after 6-month conservative treatment before consideration for surgical intervention.

\section{Exclusion Criteria}

1) The patients have associated dynamic segmental instability (Meyerding grade more than grade II) and (or) degenerative scoliosis $\left(\mathrm{Cobb} \geq 30^{\circ}\right)(14)$,

2) Lumbar spinal stenosis caused by calcification of huge lumbar disc herniation, severe DLSS and multiple-level ( $\geq 3)$ DLSS,

3) Patients who had previous back disease involving trauma, infection or pathologic causes,

4) Patients who cannot tolerate the surgery.

A total of 96 patients, 42 males and 54 females, presented with DLSS from March 2009 to December 2010. The ages ranged from 41 to 81 years (mean 62.6 years). The disease duration was 6-180 months (mean 47.1 months). According to the aforementioned classification criteria, all patients were classified as follows: clinical classification: type I 32 cases, type II 38 cases, type III 26 cases; imaging classification: type A 36 cases, type B, 60 cases, type C 0 cases; 32 patients had concurrent lumbar instability, 8 cases were associated with scoliosis $\left(\mathrm{Cobb}<30^{\circ}\right)(3)$ and 12 cases were simultaneously associated with instability and scoliosis (Table I). We chose different surgical procedures according to our classification
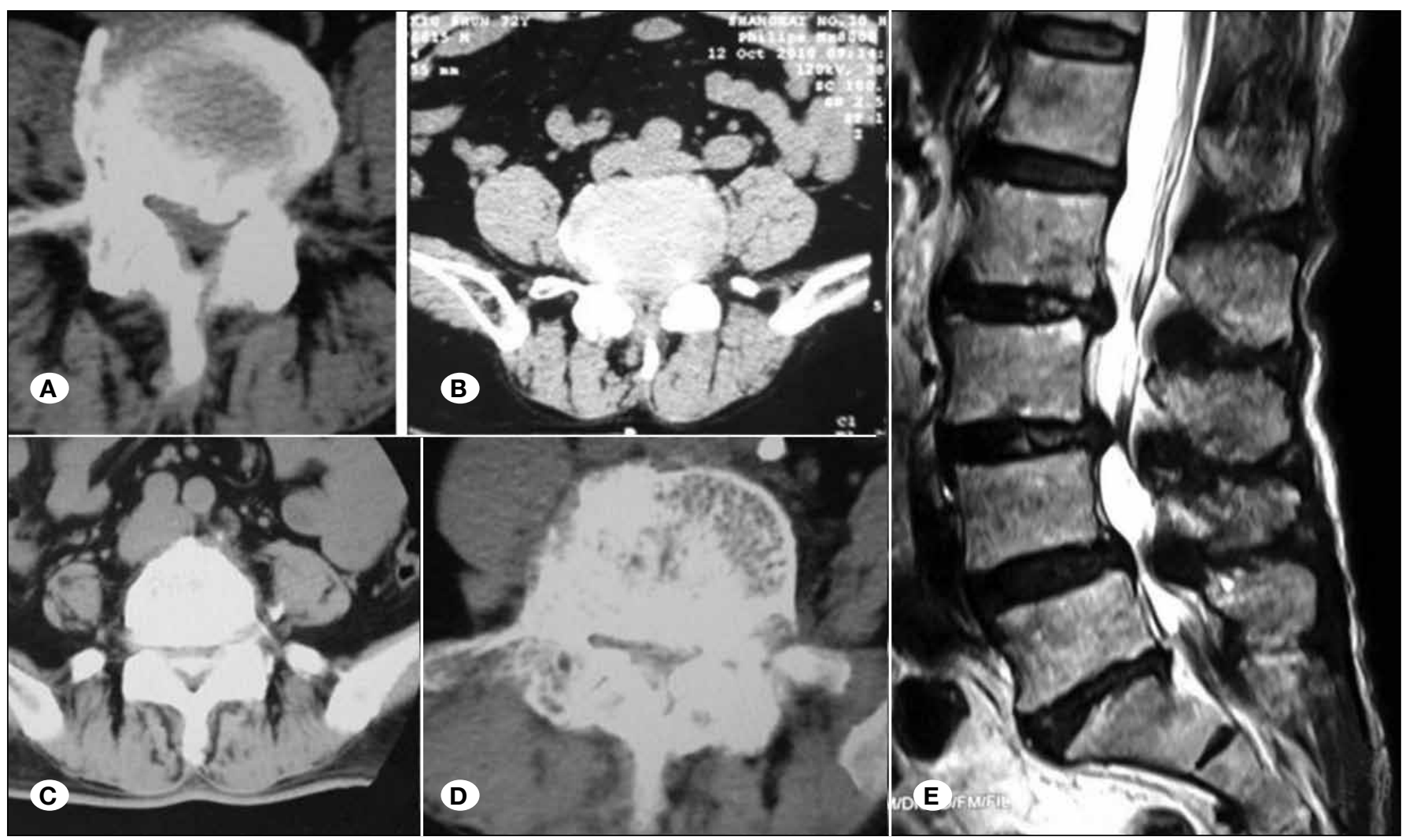

Figure 1: The patients were divided into 3 groups (type A, B, C) according to the imaging manifestation of lumbar spinal stenosis; $\mathbf{A}$ ) Type A, unilateral lateral lumbar spinal stenosis; B) Type B, bilateral lateral lumbar spinal stenosis; D) Type C, severe lumbar spinal stenosis; C) Type C, lumbar spinal stenosis caused by calcification of huge disc herniation; E) Type C, multiple-level lumbar spinal stenosis ( $\geq 3$ ). 
system (Figure 2) and all the procedures were performed by the same surgeon group. Ethical approval for this study was obtained from the Research Ethics Committee, Shanghai Tenth People's Hospital, Tongji University School of Medicine, with number 2012-RES-042. Surgical techniques were as follows:

a) Unilateral approach for bilateral decompression through an expandable tubular retractor: Under general anaesthesia, the patient was evenly placed in a prone position on a radiolucent operating table. The incision was marked by connecting a line between the outer portions of the superior and inferior pedicles (approximately $2.5 \mathrm{~cm}$ off midline) with help of a special localizer (Figure 3A-D) designed by the authors. A 3 $\mathrm{cm}$ longitudinal incision was made on the symptomatic side or the severe stenosis side according to images. After the skin and thoracolumbar fascia were dissected, the soft tissue was gradually separated by the retractor.

Adequate decompression was achieved by cutting the inferior portion of the lamina, hypertrophied superior and inferior articular processes, and ligamenta flava (12).

After one side of approach had been completely decompressed, the operating table or the expandable tubular retractor was tilted about $15^{\circ}$ to observe the contralateral side.

The basal part of the spinous process of the caudal half of the cranial lamina and a small cranial portion of the caudal lamina were removed with a rongeur. Then, the contralateral lamina was carefully removed with a rongeur leaving the ligamentum flavum in place as protection for the dural sac and the nerve root. Following sufficient resection of the bony segment, the ligamentum flavum was removed en bloc with a curette, while protecting the dural sac and contralateral nerve root with a patty. We confirmed adequate decompression of the contralateral side with recognition of the inner aspect of the pedicle on the contralateral side (15).

All procedures were accomplished under direct visualization without microscope or microendoscope.

b) Bilateral decompression via bilateral approach through an expandable tubular retractor: The decompression would be performed on the contralateral side after approach side had been finished. The details were the same as above mentioned.

c) Transforaminal lumbar interbody fusion (TLIF) and percutaneous pedicle screw fixation (PPSF) after decompression. After decompression, transforaminal lumbar interbody fusion and percutaneous pedicle screw fixation were performed through an expandable tubular retractor. Before cage insertion, the vertebral endplates were carefully prepared with disc space reamers and shavers. Sufficient autologous bone graft obtained from the resected lamina and facet was packed in the anterior disc space. A single PEEK cage (Capstone Medtronic Sofamor Danek, Memphis, TN, USA) filled with autologous bone was inserted obliquely across the disc space. No additional contralateral facet fusion was performed in the patients.

Percutaneous pedicle screws and a rod were placed on the contralateral side to distract the disc space and then the screw-rod connections in the distracted position were provisionally tightened (10).

Percutaneous pedicle screws were mounted through the Sextant system (Medtronic Sofamor Danek, Memphis, TN, USA) with the aid of a G-arm fluoroscope. The number of patients for each surgical procedure is listed in Table I.

\section{Clinical and Radiological Evaluation}

The operation time, intra-operative blood loss and complications were recorded. Radiographs (anteroposterior and lateral images) were taken 3 days after surgery to confirm the position of instruments. A CT scan was obtained from patients undergoing decompression without fixation 3 days after surgery. Out-patient follow-ups (clinical and radiological evaluation) were on the 3rd, 6th, and 12th month after surgery. Phone call follow-ups (clinical evaluation) were made every half year, 12

Table I: Demographic Data and Clinical Characteristics

\begin{tabular}{lc}
\hline Demographic data & \\
\hline Patients included & 96 \\
\hline Age, mean years (range) & $\begin{array}{c}63.4 \mathrm{yrs} \text { (range, } \\
41 \sim 81 \mathrm{yrs})\end{array}$ \\
\hline Duration of symptoms (months) & $47.1 \mathrm{~m}$ \\
\hline Clinical variables & No. patients \\
\hline Gender & 42 \\
\hline Male & 54 \\
\hline Female & 32 \\
\hline Type according to the clinical manifestations & 38 \\
\hline Type I & 26 \\
\hline Type II & \\
\hline Type III
\end{tabular}

Type according to the imagings

\begin{tabular}{cc}
\hline Type A & 36 \\
\hline Type B & 60 \\
\hline Type C & 0 \\
\hline
\end{tabular}

Concurrent with other spinal diseases

\begin{tabular}{lr}
\hline Lumbar instability & 32 \\
\hline Scoliosis & 8 \\
\hline Instability and scoliosis & 12
\end{tabular}

\begin{tabular}{lc}
\hline Surgical procedures & \\
\hline Single segment & 52 \\
\hline Procedure a & 38 \\
\hline Procedure b & 14 \\
\hline Procedure c & 28 \\
\hline Double segments & 44 \\
\hline Procedure a+ Procedure a & 28 \\
\hline Procedure a+ Procedure b & 12 \\
\hline Procedure b+ Procedure b & 4 \\
\hline Procedure c & 22 \\
\hline
\end{tabular}




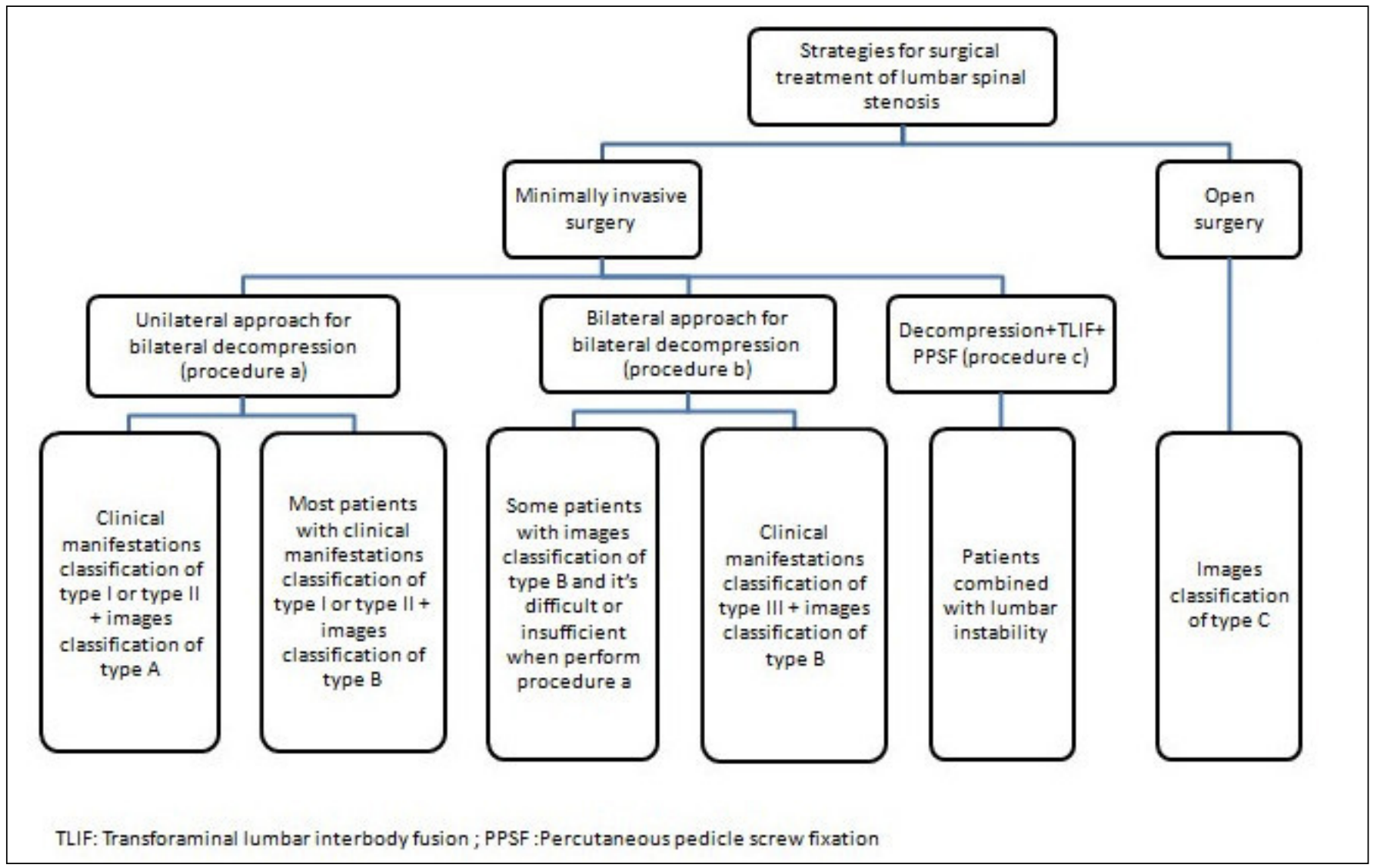

Figure 2: Surgical procedures for degenerative lumbar spinal stenosis according to the novel typing system.

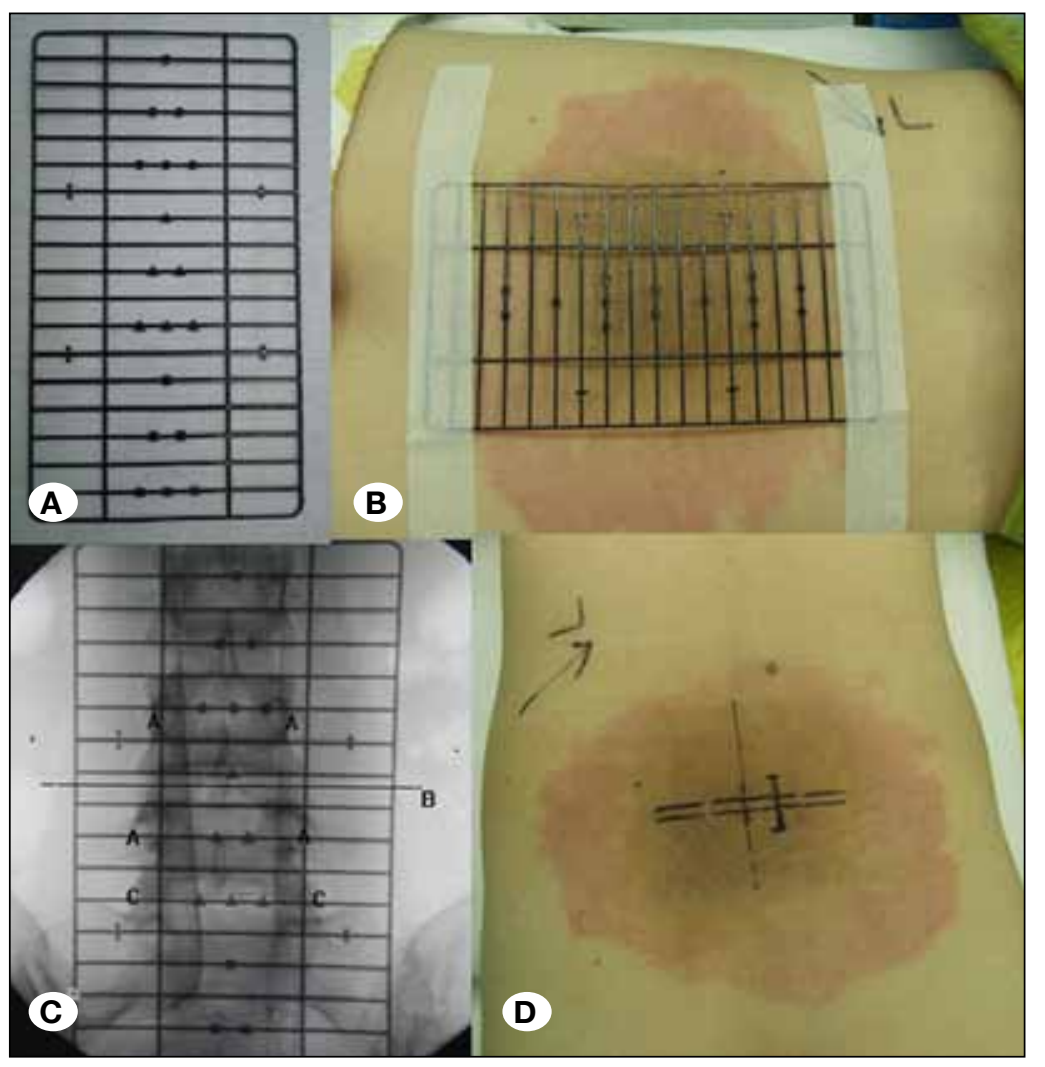

Figure 3: A) The spinal localizer consists of 19 horizontal crossbars and 4 longitudinal rods. There is about $1 \mathrm{~cm}$ between each crossbar and some different marks are made on the crossbars. Two longitudinal rods can also be distinguished with different marks. B) The locator is placed on the patient's back with the center in the midline over the approximate spinal levels of interest preoperatively. C) A represents the pedicles of $L 4$ and $L 5, B$ is the intervertebral space of L4-5, C is the superior articular process of S1.

D) The incision was marked by connecting a line between the outer portions of the superior and inferior pedicles. 
months after surgery. Visual Analogue Scale (VAS) score for low back pain and leg pain was recorded before surgery, at the 6th month after surgery and at the last follow-up respectively. The Oswestry disability index (ODI) was used to assess how the patient's leg (or back) trouble had affected the ability to manage in daily life. The clinical improvement rate was assessed by the Japanese Orthopaedic Association (JOA) score with a maximum possible score of 29 points pre- and postoperatively. [The JOA improvement rate was calculated as (post-operative JOA scores- preoperative JOA scores) / (29- preoperative JOA scores) $\times 100 \%$. Operation efficacy was then determined by the clinical improvement rate as excellent $\geq 75 \%, 50 \% \leq$ good $<75 \%, 25 \% \leq$ fair $<50 \%$, and poor $<25 \%$ or postoperative JOA scores lower than preoperative scores. On the 3rd, 6th, 12th months after surgery, radiographs (anteroposterior and lateral images) were obtained to evaluate the fusion status. A CT scan with two-dimensional reconstruction was performed when it was difficult to confirm the fusion status by radiographs.

2 independent, experienced spine surgeons, who were not part of the surgical team, performed the radiographic assessment of fusion. The fusion status was assessed according to Bridwell's posterior fusion grades using plain radiographs $(2,4)$, namely, grade I: solid trabeculated transverse process and facet fusions bilaterally, grade II: thick fusion mass on one side, difficult to visualize on the other side, grade III: suspected lucency or defect in the fusion mass, grade IV: definite resorption of graft with fatigue of instrumentation. The fusion was defined as grade I or grade II.

The software of SPSS 17.0 (SPSS, USA) was used to statistical analysis. Quantifiable data were expressed as $\bar{X} \pm s$. Student's $t$ test was used for the comparison of continuous variables. $P$ values below 0.05 were accepted for significance.

\section{RESULTS}

The operation time and intra-operative blood loss are illustrated in Table II. In 1 patient who also had severe osteoporosis, the interbody cage sunk into the upper vertebral body. The cage was removed and the interbody space was then fused with autologous bone graft. Dural tear occurred in 1 patient. The overlying fascia was closed tightly without additional exposure and repair in this patient. Postoperatively, the patients remained strictly supine in bed and cerebrospinal fluid leakage lasted 4 days without any neurological sequelae or wound complication. Delayed healing of the incision was found in 3 cases, which was considered to be a result of excessive traction during the operation. The wound healed 15 18 days after surgery with antibiotics and dressing change. The mean follow-up was 24 months with a range of 15 36 months. The VAS score of low back pain before surgery was $(6.6 \pm 1.0)$, and $(2.8 \pm 0.7)$ and $(2.6 \pm 0.7)$ at six months after surgery and the last follow-up respectively. The VAS score of leg pain before surgery was $(7.8 \pm 1.0)$, and were $(2.3 \pm 0.7)$ and $(2.0 \pm 0.7)$ at six months and the last follow-up respectively. ODI (\%) were $(67.7 \pm 6.5),(19.0 \pm 2.7)$ and $(16.8 \pm 1.7)$ before surgery, at six months after surgery, and the last follow-up respectively. VAS score and ODI showed statistically significant improvements after surgery $(P<0.05)$. JOA scores before surgery and the last follow-up after surgery were $(7.9 \pm 1.7)$ and $(24.3 \pm 1.7)$ respectively. According to the JOA scores, the operation efficacy was excellent in 57 cases, good in 36 cases, and fair in 3 cases. 50 patients who also had lumbar instability underwent transforaminal lumbar interbody fusion and percutaneous pedicle screw fixation after decompression (Figure 4A-O). Postoperative radiographs showed one pair of screws was overlong and penetrating the front cortical bone of the vertebra in 1 patient (Figure 5A,B), and no additional symptoms (such as abdominal pain) were observed during the follow-up period in this patient. According to the Bridwell's criterion, there were 36 grade I cases (72\%), 12 grade II cases(24\%), and 2 grade III cases (4\%). The patients of grade III are still in follow up.

\section{DISCUSSION}

Spinal stenosis is a narrowing of the spinal canal by a combination of bone and soft tissues, which causes mechanical compression of spinal nerve roots. The compression of these nerve roots can be asymptomatic, but it can also become symptomatic, resulting in weakness, reflex alterations, gait disturbances, bowel or bladder dysfunction, motor and sensory changes, radicular pain or atypical leg pain, and neurogenic claudication $(1,13)$. DLSS typically starts with a normal canal and changes such as bulging discs, zygapophyseal joint hypertrophy, buckling or hypertrophy of the ligaments, and spondylolisthesis develop as a result of

Table II: Operation Time (min) and Intra-Operative Blood Loss $(\mathrm{ml})$ of the Procedures

\begin{tabular}{|c|c|c|c|c|c|c|c|}
\hline & \multicolumn{3}{|c|}{ Single segment $(n=52)$} & \multicolumn{4}{|c|}{ Double segments $(n=44)$} \\
\hline & $\begin{array}{l}\text { Procedure a } \\
\qquad(n=38)\end{array}$ & $\begin{array}{l}\text { Procedure b } \\
(n=14)\end{array}$ & $\begin{array}{c}\text { Procedure c } \\
(n=28)\end{array}$ & $\begin{array}{c}\text { Procedure a } \\
+ \text { Procedure } \\
a \\
(n=28)\end{array}$ & $\begin{array}{c}\text { Procedure a } \\
+ \text { Procedure } \\
b \\
(n=12)\end{array}$ & $\begin{array}{c}\text { Procedure b } \\
+ \text { Procedure } \\
\text { b } \\
(n=4)\end{array}$ & $\begin{array}{c}\text { Procedure c } \\
(n=22)\end{array}$ \\
\hline Operation time (min) & $55.4 \pm 15.2$ & $87.1 \pm 14.6$ & $136.4 \pm 26.5$ & $102.1 \pm 16.6$ & $118.3 \pm 18.0$ & $153.8 \pm 18.9$ & $178.6 \pm 24.5$ \\
\hline $\begin{array}{l}\text { Intra-operative blood } \\
\text { loss (ml) }\end{array}$ & $88.0 \pm 44.0$ & $142.9 \pm 53.1$ & $168.0 \pm 77.2$ & $176.4 \pm 71.3$ & $216.3 \pm 75.2$ & $312.5 \pm 62.9$ & $253.2 \pm 74.1$ \\
\hline
\end{tabular}

Note: Procedure a: Unilateral approach for bilateral decompression; procedure b: Bilateral approach for bilateral decompression; procedure c: Transforaminal lumbar interbody fusion (TLIF) and percutaneous pedicle screw fixation (PPSF) after decompression. 


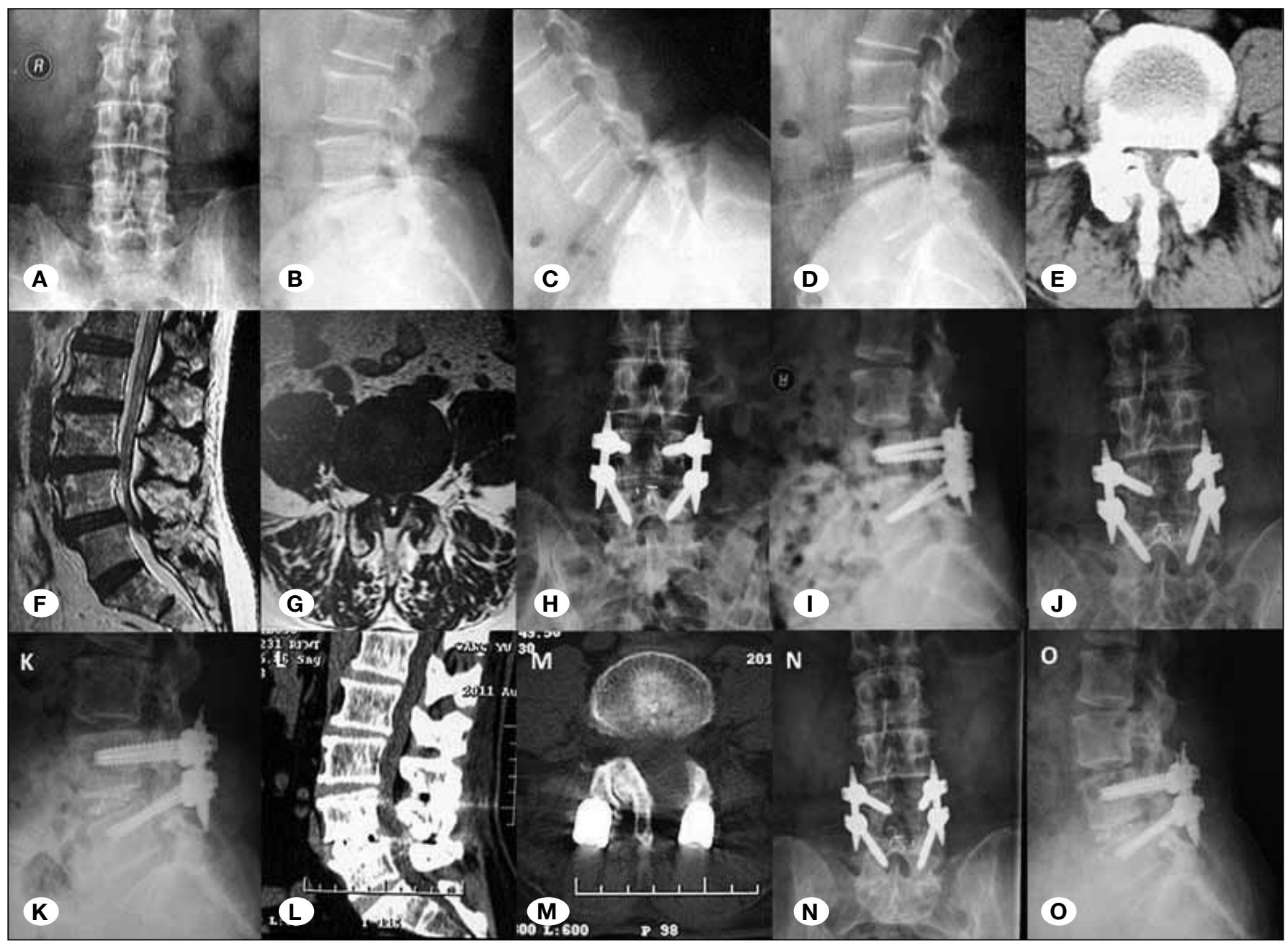

Figure 4: A 62-year-old man was admitted to our hospital for low back pain and intermittent claudication for almost 2 years. According to our classification system, he was classified as type II for clinical manifestations and type B for imaging features. The patient also had lumbar instability. We chose the unilateral approach for bilateral decompression, transforaminal lumbar interbody fusion and percutaneous pedicle screw fixation. A, B, C, D, E, F, G) The preoperative image data (X-ray, CT and MRI) showed lumbar stenosis combined with instability in L4/5 level. $\mathbf{H}$, I) AP and lateral radiographs showing the internal fixation and cage on the third day after surgery; J, K) Three months after surgery; L, M) CT scan showing a solid fusion and satisfactory decompression six months after surgery; N, O) AP and lateral radiographs showing a solid fusion 12 months after surgery.

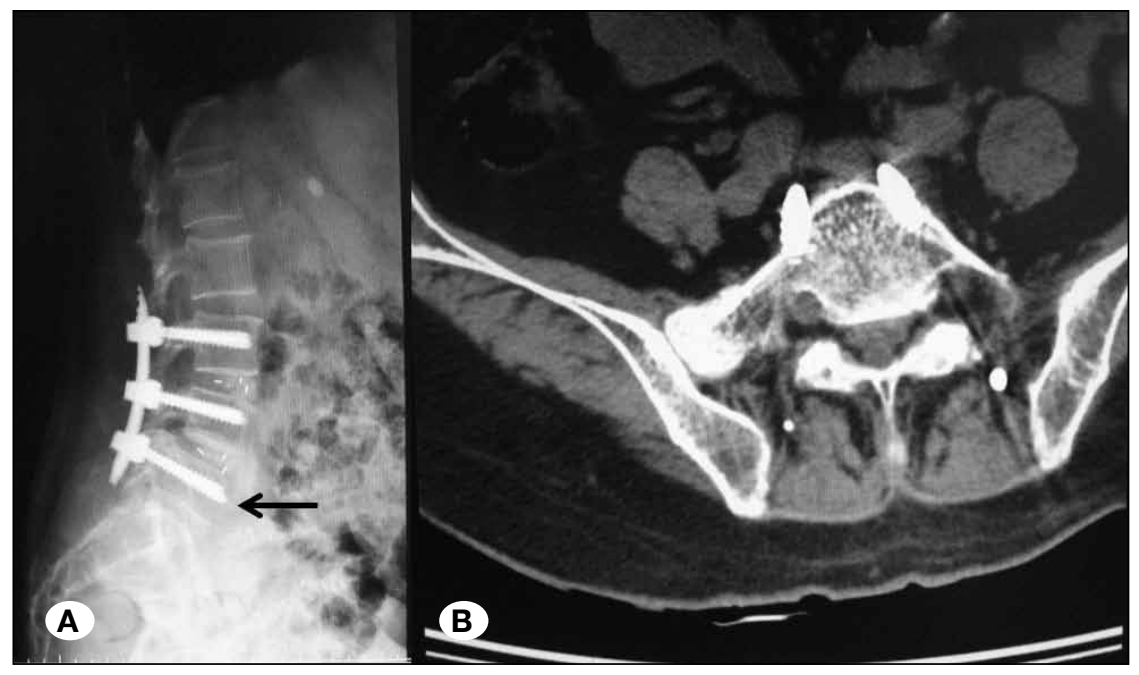

Figure 5: Postoperative $X$ ray and CT showed one pair of screws was overlong and penetrating the front cortical bone of the vertebra in 1 patient in group $A$. 
Gu G. et al: Minimally Invasive Treatment of Lumbar Spinal Stenosis

the degenerative cascade. DLSS can anatomically involve the central canal, lateral recess, foramina or any combination of these locations. Central canal stenosis may result from a decrease in the anteroposterior, transversal or combined diameter secondary to loss of disc height with or without ligamentum flavum buckling or hypertrophy, disc protrusion, hypertrophic zygapophyseal joints, and degenerative spondylolisthesis (5). The same processes, including decreased disc height, facet joint hypertrophy (with or without spondylolisthesis) and/or vertebral endplate osteophytosis, can also result in lateral recess stenosis. Foraminal stenosis can be either antero-posterior resulting from a combination of disc-space narrowing and overgrowth of structures anterior to the facet joint capsule, and/or vertical resulting from posterolateral osteophytes from the vertebral endplates protruding into the foramen along with a laterally bulging annulus fibrosis or herniated disc that compresses the nerve root against the superior pedicle (7). The pathoanatomy of spinal stenosis is very complicated, and how to choose minimally invasive surgical procedures for lumbar spinal stenosis is still controversial. Our suggestions (Figure 5) are as follows: unilateral approach for bilateral decompression applies to patients with a clinical manifestations classification of type I or type II and associated with an images classification of type A or most of type B. However, in certain cases with an images classification of type $B$, the contralateral decompression should be performed if unilateral approach for contralateral decompression was difficult or insufficient. For patients with clinical manifestations classification of type I or type III and images classification of type B, a bilateral approach for bilateral decompression was necessary. For patients with images classification of type $\mathrm{C}$, open surgery could be a safe and optimal option. For those patients who also had lumbar instability (6), transforaminal lumbar interbody fusion and percutaneous pedicle screw fixation should be performed after decompression. Patients with combined scoliosis should be treated as degenerative scoliosis if the Cobb angle is $\geq$ $30^{\circ}$. If the Cobb angle is $<30^{\circ}$ without symptoms of instability, the patients could be treated as simple DLSS but it should be noted that articular facets on the concave side should be carefully preserved. In some cases, internal fixation and short level fusion were needed to prevent aggravation of scoliosis and instability. We performed contralateral decompression after adequate decompression approach side had been achieved. The operating table or the expandable tubular retractor was tilted about $15^{\circ}$ to observe the contralateral side. The root of spinous process was partly excised to visualize the contralateral structures. In order to protect the dural sac and nerve root, we first decompressed to the contralateral pedicle beyond the surface of ligamentum flavum. The bilateral approach for bilateral decompression should be performed if the unilateral approach for contralateral decompression was difficult or insufficient. For patients combined with preoperative lumbar instability or intraoperative iatrogenic instability during operation, minimally invasive transforaminal lumbar interbody fusion (MIS- TLIF) should be performed. Currently, numerous studies $(8,11)$ have reported that MIS-TLIF was similar to open TLIF in respect of long-term clinical outcomes and fusion rates. However, MIS- TLIF has the additional benefits of less postoperative pain, early rehabilitation, shorter hospitalization, and fewer complications. Study by Shunwu et al. (12) showed that serum creatine kinase was significantly lower in the MISTLIF group than in the open group on the third postoperative day and the average postoperative ODI and VAS scores were significantly reduced in both groups. However, the scores in the minimally invasive group improved more compared with the open group.

In our study, VAS scores and ODI after surgery were both significantly improved as well. According to the JOA scores, the operation efficacy was excellent in 57 cases, good in 36 cases, fair in 3 cases. According to the Bridwell's criterion, the fusion rate was $96 \%(48 / 50)$ in patients who underwent fusion surgery. There were no complications related to the cages or pedicle screws. There were a few limitations of this study. First, the number of cases included in this study was relatively low. Second, this was a retrospective study without any control group, and the potential interference factors such as the surgeon's habits, the operation conditions and so on could not be excluded. Third, postoperative CT scans were not performed in all patients, and deviations may have existed when assessing the fusion status. Further studies are needed to make clear that minimally invasive surgery for DLSS associated with severe instability, with degenerative scoliosis $\left(\right.$ Cobb $\geq 30^{\circ}$ ), lumbar spinal stenosis caused by calcification of huge lumbar disc herniation, and multiple-level $(\geq 3)$ DLSS is beneficial.

\section{- CONCLUSION}

Minimally invasive surgical treatment of DLSS has satisfactory outcomes according to the novel classification, but further long-term, prospective, randomized controlled studies involving a larger study group are needed to validate the long-term efficacy.

\section{ACKNOWLEDGEMENT}

We thank Xia Zhang, a nurse in our hospital, for her excellent daily work in our surgery.

\section{REFERENCES}

1. Botwin KP, Gruber RD: Lumbar spinal stenosis: Anatomy and pathogenesis. Phys Med Rehabil Clin N Am 14:1-15, 2003

2. Bridwell $\mathrm{KH}$, Lenke LG, McEnery KW, Baldus C, Blanke K: Anterior fresh frozen structural allografts in the thoracic and lumbar spine. Do they work if combined with posterior fusion and instrumentation in adult patients with kyphosis or anterior column defects? Spine 20:1410-1418, 1995

3. Costa F, Sassi M, Cardia A, et al: Degenerative lumbar spinal stenosis: Analysis of results in a series of 374 patients treated with unilateral laminotomy for bilateral microdecompression. J Neurosurg Spine 7:579-586, 2007

4. Eck KR, Lenke LG, Bridwell KH, Gilula LA, Lashgari CJ, Riew KD: Radiographic assessment of anterior titanium mesh cages. $J$ Spinal Disord 13(6):501-509, 2000

5. Genevay S, Atlas SJ: Lumbar spinal stenosis. Best Pract Res Clin Rheumatol 24(2):253-265, 2010 
6. Hanley EN Jr: The indications for lumbar spinal fusion with and without instrumentation. Spine 20:143S-153S, 1995

7. Jenis LG, An HS: Spine update. Lumbar foraminal stenosis. Spine 25(3):389-394, 2000

8. Lau D, Lee JG, Han SJ, Lu DC, Chou D: Complications and perioperative factors associated with learning the technique of minimally invasive transforaminal lumbar interbody fusion (TLIF). $\mathrm{J}$ Clin Neurosci 18:624-627, 2011

9. Manchikanti L, Cash KA, McManus CD, Pampati V, Abdi $\mathrm{S}$ : Preliminary results of a randomized, equivalence trial of fluoroscopic caudal epidural injections in managing chronic low back pain: Part 4--Spinal stenosis. Pain Physician 11:833-848, 2008

10. Park Y, Ha JW, Lee YT, Oh HC, Yoo JH, Kim HB: Surgical outcomes of minimally invasive transforaminal lumbar interbody fusion for the treatment of spondylolisthesis and degenerative segmental instability. Asian Spine J 5(4):228-236, 2011
11. Peng CW, Yue WM, Poh SY, Yeo W, Tan SB: Clinical and radiological outcomes of minimally invasive versus open transforaminal lumbar interbody fusion. Spine 34:1385-1389, 2009

12. Shunwu F, Xing Z, Fengdong Z, Xiangqian F: Minimally invasive transforaminal lumbar interbody fusion for the treatment of degenerative lumbar diseases. Spine (Phila Pa 1976) 35(17):16151620, 2010

13. Simotas AC, Dorey FJ, Hansraj KK, Cammisa F Jr: Nonoperative treatment for lumbar spinal stenosis. Clinical and outcome results and a 3-year survivorship analysis. Spine (Phila Pa 1976) 25:197203, 2000

14. Tribus CB: Degenerative lumbar scoliosis: Evaluation and management. J Am Acad Orthop Surg 11:174-183, 2003

15. Yang SM, Park HK, Chang JC, Kim RS, Park SQ, Cho SJ: Minimum 3-year outcomes in patients with lumbar spinal stenosis after bilateral microdecompression by unilateral or bilateral laminotomy. J Korean Neurosurg Soc 54(3):194-200, 2013 